\title{
An interesting case of lipoid pneumonia
}

lasonas Dermitzakis ${ }^{1}$, Drosos Tsavlis², Polyanthi Konstantinidou ${ }^{1,3}$, Charalampos Charalampidis ${ }^{4}$, Eleni Zagelidou ${ }^{3}$, Doxakis Anestakis ${ }^{1,3,4}$

\section{Dear Editor,}

Pneumonia is an inflammatory disease of the lung parenchyma in which alveoli are filled with fluid and blood cells ${ }^{1}$. This condition is usually caused by viral or bacterial infections of the lung tissue, while it may also be due to aspiration of vomit or food ${ }^{2}$. Although the most common form of pneumonia is bacterial, lipoid pneumonia (LP) is one of the most difficult to diagnose.

The case of a 44-year-old white male with unknown medical history was investigated to determine the exact cause of death. The patient was found dead and transferred to the Forensic Medical Service of Thessaloniki, where an autopsy was performed. A biopsy was done on the deceased's three key organs (heart, liver and lungs), and the most important finding was related to the lung tissue sample. We observed foci of fibrous connective tissue, swelling in specific areas, regions of hemorrhagic infiltration, plus alveoli and bronchioles destruction due to lipid deposition (Figure 1). These findings led us to consider LP as the cause of death.

$L P$ is an infectious disease of the lungs that results from entering fat into the bronchial tree ${ }^{3}$. There are two types of LP: exogenous and endogenous ${ }^{4}$. The exogenous LP occurs when the fat particles enter from outside the body and reach the lungs via the nose or mouth ${ }^{5}$. The endogenous LP occurs in airway obstruction. In this case, fatty particles are released from the cellular breakdown in alveolar walls, and the macrophages, which phagocytose these lipids, fill the lumen of the disconnected airspace ${ }^{6}$. An ill-defined, pale-yellow area on the lung reflects the gross appearance of LP. At the microscopic scale, foamy macrophages and giant cells are seen in the airways, and the inflammatory response is visible in the parenchyma? ${ }^{7}$.

In our cause, a young drug abuser died due to LP. Because of the patient's unknown medical history, we cannot confidently assume why the young man's situation led to a manifestation of LP. Nevertheless, we know that the patient was a drug addict, an essential element if we consider recent studies on pulmonary diseases of drug addicts ${ }^{8}$. The main reason that could lead to this case of LP is overdose. Usually, overdose leads to coma (stage 1, 2 or 3$)^{9}$. In these stages of coma, aspiration is regularly caused due to lack

\section{AFFILIATION}

1 Department of Autopsy Histopathology, Laboratory of Forensic Medicine and Toxicology, School of Medicine, Aristotle University of Thessaloniki, Thessaloniki, Greece

2 Laboratory of Experimental Physiology, School of Medicine, Aristotle University of Thessaloniki, Thessaloniki, Greece 3 Laboratory of Pathology, Forensic Medical Service of Thessaloniki, Thessaloniki, Greece 4 Department of Anatomy, Medical School of the University of Cyprus, Nicosia, Cyprus

\section{CORRESPONDENCE TO}

Doxakis Anestakis. Laboratory of Pathology, Forensic Medical Service of Thessaloniki, Diavata, 57008, Thessaloniki, Greece. E-mail: path.autopsylab@gmail.com ORCID ID: https://orcid.org/0000-0001-8004-7666

\section{KEYWORDS}

lipoid pneumonia (LP), respiratory diseases, drug abuser

Received: 24 August 2021

Accepted: 20 October 2021
Figure 1. Lipoid material, inflammatory cells and young fibroblasts (H/E a.X100, b.X200)
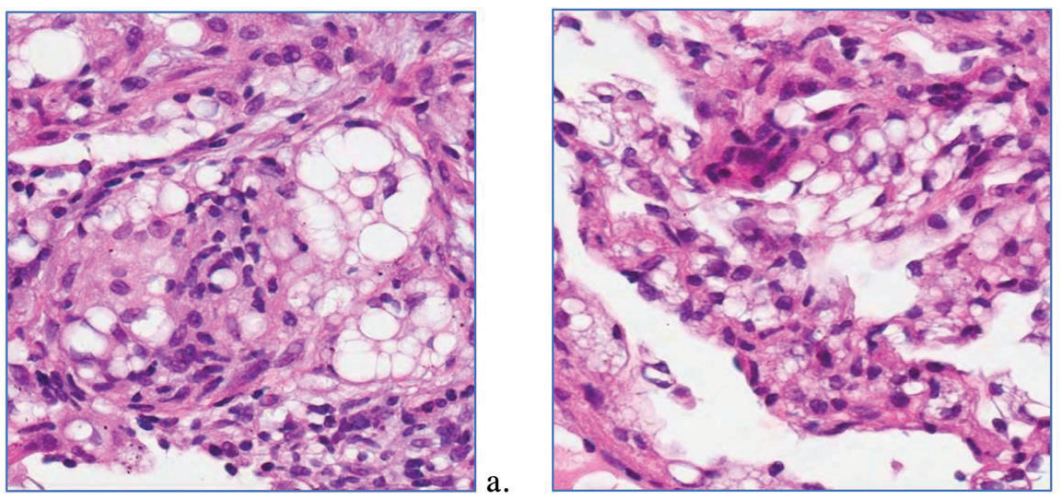

b. 
of consciousness ${ }^{10}$. For this reason, aspiration of gastric contents into the lungs is often associated with a drug overdose.

In summary, LP can be challenging to diagnose. Most types of pneumonia are visible on a chest X-ray. However, a chest $X$-ray is not enough to identify which type of pneumonia patients have. Owing to the nonspecific clinical presentation and radiological features, the diagnosis is often missed or delayed. As seen in our case, LP led to the sudden death of the young patient. Consequently, diagnosis of this disease requires a high index of suspicion and can be confirmed by demonstrating lipid-laden macrophages in respiratory samples such as sputum, bronchoalveolar lavage fluid or fine-needle aspiration biopsy from lung lesions.

\section{CONFLICTS OF INTEREST}

The authors have completed and submitted the ICMJE Form for Disclosure of Potential Conflicts of Interest and none was reported.

\section{FUNDING}

There was no source of funding for this research.

\section{ETHICAL APPROVAL AND INFORMED CONSENT}

Ethical approval and informed consent were not required for this study.

\section{DATA AVAILABILITY}

The data supporting this research cannot be made available for privacy reasons.

\section{PROVENANCE AND PEER REVIEW}

Not commissioned; internally peer reviewed.

\section{REFERENCES}

1. Hall JE. Guyton and Hall Textbook of Medical Physiology. 13th ed. Saunders; 2015.

2. Sherwood L. Introduction to human physiology. 8th ed. Brooks/Cole; 2013.

3. Pelz L, Hobusch D, Erfurth F, Richter K. Familiäre Cholesterinpneumonie. Familial cholesterol pneumonia. Helv Paediatr Acta. 1972;27(4):371-379.

4. Bell MM. Lipoid pneumonia: An unusual and preventable illness in elderly patients. Can Fam Physician. 2015;61(9):775-777. Accessed August 24, 2021. https:// www.cfp.ca/content/cfp/61/9/775.full.pdf

5. Kim JY, Jung JW, Choi JC, Shin JW, Park IW, Choi BW. Recurrent lipoid pneumonia associated with oil pulling. Int J Tuberc Lung Dis. 2014;18(2):251-252. doi:10.5588/ijtld.13.0852

6. Antoon JW, Hernandez ML, Roehrs PA, Noah TL, Leigh MW, Byerley JS. Endogenous lipoid pneumonia preceding diagnosis of pulmonary alveolar proteinosis. Clin Respir J. 2016;10(2):246-249. doi:10.1111/crj.12197
7. Lipid pneumonia. Wikipedia. Updated August 2, 2021. Accessed September 14, 2021. https://en.wikipedia.org/ wiki/Lipid_pneumonia

8. Gottlieb LS, Boylen TC. Pulmonary complications of drug abuse. West J Med. 1974;120(1):8-16. Accessed August 24, 2021. https://www.ncbi.nlm.nih.gov/pmc/articles/ PMC1129296/pdf/westjmed00305-0030.pdf

9. Sapira JD, McDonald RH Jr. Drug Abuse-1970. Dis Mon. 1970;16(11):1-47. doi:10.1016/S0011-5029(70)80015-3

10. Huxley EJ, Viroslav J, Gray WR, Pierce AK. Pharyngeal aspiration in normal adults and patients with depressed consciousness. Am J Med. 1978;64(4):564-568. doi:10.1016/0002-9343(78)90574-0 